\title{
BIFURCATION OF SPATIAL CENTRAL CONFIGURATIONS FROM PLANAR ONES*
}

\author{
RICHARD MOECKEL ${ }^{\dagger}$ AND CARLES SIMÓ ${ }^{\ddagger}$
}

\begin{abstract}
Central configurations are important special solutions of the Newtonian $N$-body problem of celestial mechanics. In this paper a highly symmetrical case is studied. As the masses are varied, spatial central configurations appear through bifurcation from planar ones. In particular, spatial configurations can be found which are arbitrarily close to being planar.
\end{abstract}

Key words. celestial mechanics, central configurations, bifurcation

AMS subject classifications. $34,58,70,85$

1. Introduction. This paper is concerned with certain highly symmetrical central configurations of the Newtonian $N$-body problem. It is shown that under certain conditions, spatial central configurations (that is, nonplanar ones) bifurcate from planar ones as the masses are varied. Moreover, the bifurcation is described in detail.

Recall that the $N$-body problem concerns the motion of $N$ point particles with masses $m_{j} \in \mathbf{R}^{+}$and positions $q_{j} \in \mathbf{R}^{3}$, where $j=1, \ldots, N$. The motion is governed by Newton's law

$$
m_{j} \ddot{q}_{j}=\frac{\partial U}{\partial q_{j}}
$$

Here $U(q)$ is the Newtonian potential

$$
U(q)=\sum_{\substack{(i, j) \\ i<j}} \frac{m_{i} m_{j}}{\left|q_{i}-q_{j}\right|} .
$$

Let $q=\left(q_{1}, \ldots, q_{N}\right)^{T} \in \mathbf{R}^{3 N}$ and $M=\operatorname{diag}\left(m_{1}, m_{1}, m_{1}, \ldots, m_{N}, m_{N}, m_{N}\right)$. Then the equation of motion can be written as follows:

$$
\ddot{q}=M^{-1} \frac{\partial U}{\partial q} .
$$

In studying this problem, there is no loss of generality in assuming that the center of mass of the particles is at the origin: $m_{1} q_{1}+\cdots+m_{N} q_{N}=0$. Because the potential is singular when two particles have the same position, it is natural to assume that the configuration avoids the set $\Delta=\left\{q: q_{i}=q_{j}\right.$ for some $\left.i \neq j\right\}$.

DEFINITION 1. A configuration $q \in \mathbf{R}^{3} \backslash \Delta$ is called a central configuration if there is some constant $\lambda$ such that

$$
M^{-1} \frac{\partial U}{\partial q}=\lambda q
$$

* Received by the editors May 3, 1993; accepted for publication (in revised form) November 29, 1993.

† School of Mathematics, University of Minnesota, Minneapolis, Minnesota 55455. This research was supported by the National Science Foundation, the Sloan Foundation, and the Eidgenösische Technische Hochschule, Zürich.

‡ Departament de Matemàtica Aplicada i Anàlisi, Universitat de Barcelona, Gran Via 585, 08007 Barcelona, Spain. This research was partially supported by CICYT grant ESP91-403. 